\title{
The purple urine bag syndrome: a visually striking side effect of a highly alkaline urinary tract infection
}

\author{
Peter Peters, BSc (Hons), MBBS, ; ${ }^{*}$ Jessica Merlo, BSc, MBBS, MBA; ${ }^{*}$ Nicholas Beech, $B S c_{;}{ }^{\dagger}$ Chantelle Giles, \\ $B S c ;{ }^{\dagger}$ Bonita Boon, RN;; Brian Parker, BAppSc; ${ }^{\ddagger}$ Craig Dancer, MBBS, FRACP, FRCPA; ${ }^{\ddagger}$ Wendy Munckhof, \\ MBBS, FRACP, FRCPA, PhD; ;ł H.S. Teng, MBBS(Hons), FRACS(Urol)*
}

*Department of Urology, Ipswich General Hospital, Queensland, Australia; †School of Medicine, University of Queensland, Queensland, Australia; ${ }^{\S}$ School of Medicine, Griffith University, Queensland, Australia; ¥Microbiology, Queensland Health Pathology Services, Ipswich General Hospital, Queensland, Australia; Infectious Diseases, Ipswich General Hospital, Queensland, Australia

Cite as: Can Urol Assoc J 2011;5(4):233-4; D01:10.5489/cuaj.10177

7 he purple urine bag is a visually striking and rarely seen side effect of a urinary tract infection. The change in colour is purely within the bag itself; interestingly, the urine itself remains unchanged in colour.

Our patient, an 82-year-old female, had a long-standing

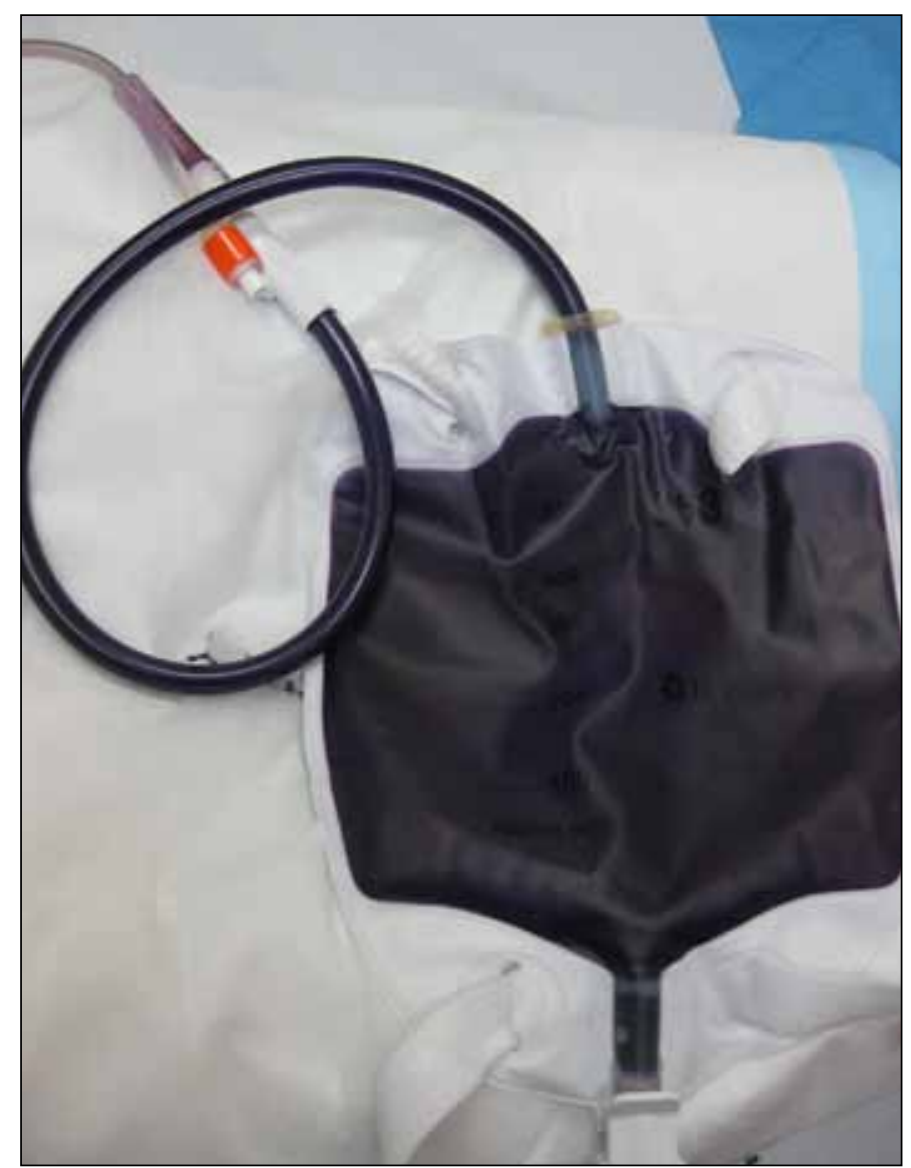

Fig. 1. The urine bag still attached to the removed supra pubic catheter. Colour change in consistent throughout the bag and extends (red in colour) into the catheter. supra pubic catheter (SPC) in situ; she presented for her routine 6 -week SPC change. During this visit, the colour change of the urine drainage bag was noted. The change in colour was consistent throughout the bag and extended to the urinary catheter, although not in the same shade of deep purple noted in the drainage bag (Fig. 1). The urine from the bag contained sediment which was sent for analysis.

The urine contained within the purple drainage bag was not purple on visual examination (Fig. 2), although the urine collected following the re-insertion of a new SPC was noticeably lighter in colour. There was no hindrance to the removal of the old SPC, although the patient complained of mild discomfort upon removal. The new SPC was inserted without difficulty. Our patient denied any recent history of nausea, fevers, sweats, chills or vomiting. The patient also denied any recent changes to her medication regimen or unusual dietary changes.

Urinary dipstick analysis indicated the presence of large leucocytes, positive nitrates and a $\mathrm{pH}$ of 8.5. Following

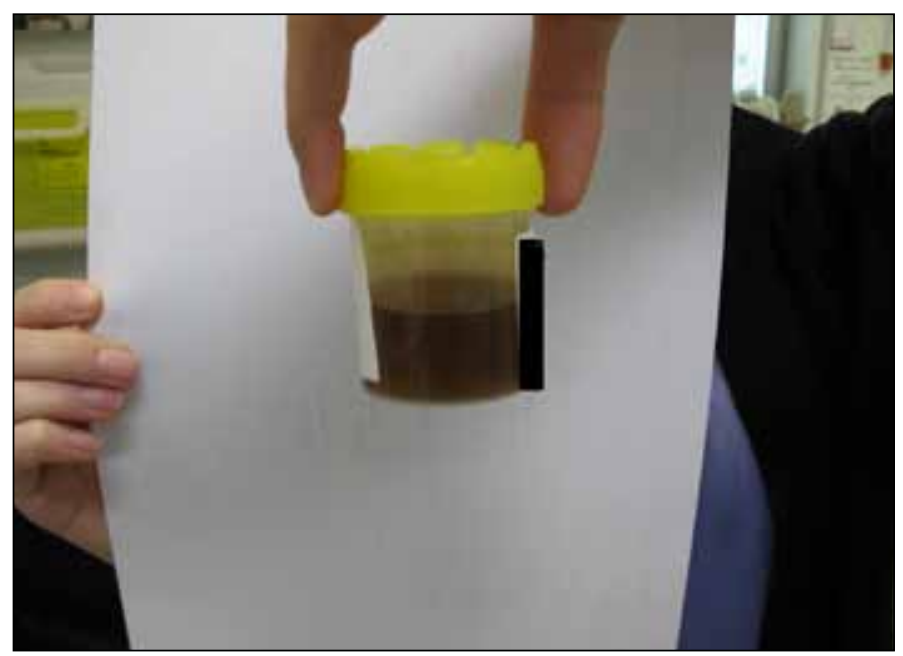

Fig. 2. Urine collected from the purple urine bag. The urine itself was dark in colour but not purple in colour. 


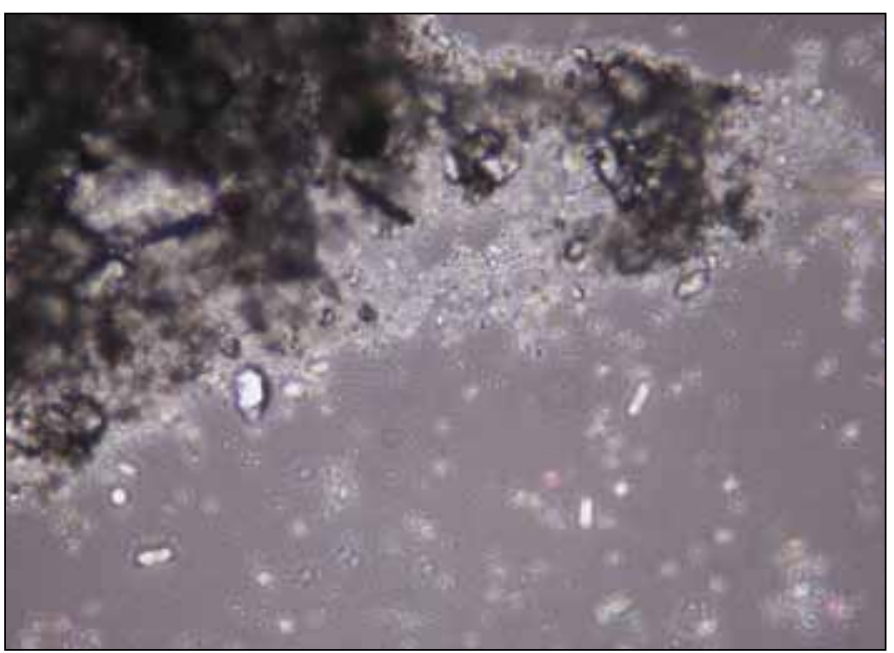

Fig. 3. Microscopy of the sediment collected from the urine sample within the purple urine bag, power of 10 , indicating phosphate crystals.

consultation with our hospitals infectious diseases team, the patient started on a course of amoxicillin/clavulanic acid 875/125 mg BD for 5 days, while urine microscopy/ culture/sensitivity (M/C/S) was conducted. Culture revealed the presence of Proteus mirabilis, sensitive to amoxicillin/ clavulanic acid. Microscopy revealed the presence of phosphate crystals within the sediment from the purple urine bag (Fig. 3), while a smaller volume of phosphate crystals were noted in the second batch of urine from the fresh catheter/collection bag. The presence of phosphate crystals is a known side effect of a Proteus mirabilis infection.

Based upon the patient's history and the microbiology results, a diagnosis of purple urine bag syndrome was made. This is a rarely reported state where the urine collection bag is noted to change to purple. The condition is most common in patients with permanent urinary catheterization, either urethral or supra pubic, in combination with a highly alkaline urinary tract infection (UTI). Numerous organisms have been reported as being responsible for the purple urine bag and include Providencia bacteria, Escherichia coli, Proteus mirabilis or Klebsiella pneumonie. ${ }^{1,2}$

The pathogenesis of purple urine bag syndrome is due to the metabolism of tryptophan by bacteria to indole and later converted to indicant in the liver. ${ }^{3}$ This is excreted and broken down in the urine by bacteria possessing one or both enzymes, sulphatise and phosphatase that metabolize this pigment to indirubin and indigo in an alkaline environment (urine).

The exact cause of the colour change in purple urine bag syndrome is still unclear. Dealler and colleagues suggest it is due to tryptophan being metabolized to indole which is absorbed into the portal system and converted to indicant by the liver. ${ }^{1,3}$ This, in turn, is excreted into the urine where the presence of an alkaline environment and bacteria are capable of metabolizing indicant to indirubin and indigo. ${ }^{1,3}$ The indigo can also be present in the catheter itself, giving a blue discolouration. ${ }^{4}$

While purple urine bag syndrome may seem harmless, the underlying UTI can lead to serious consequences in a patient with a long-term urinary catheter in which the standard warning signs of dysuria may not be apparent on top of other medical comorbidities. Medical management of purple urine bag syndrome does not require any special treatment apart from changing the catheter and administering appropriate antimicrobial therapy to treat the underlying bacterial infection.

Competing interests: None declared.

This paper has been peer-reviewed.

\section{References}

1. Lin $\mathrm{CH}$, Huang $\mathrm{HT}$, Chien $\mathrm{CC}$, et al. Purple urine bag syndrome in nursing homes: ten elderly case reports and a literature review. Clin Interv Aging 2008;3:729-34.

2. Tan CK, Wu YP, Wu HY, et al. Purple urine bag syndrome. CMAJ 2008;179:491.

3. Dealler SF, Hawkey PM, Millar MR. Enzymatic degradation of urinary indoxyl sulfate by Providencia stuartii and Klebsiella pneumoniae causes the purple urine bag syndrome. J Clin Microbiol 1988;26:2152-6.

4. Harun NS, Nainar SK, Chong VH. Purple urine bag syndrome: a rare and interesting phenomenon. South Med J 2007;100:1048-50.

Correspondence: Dr. Peter Peters, 43 Shelduck Pl, Calamvale, Q 4116, Australia; peter2734@gmail.com 